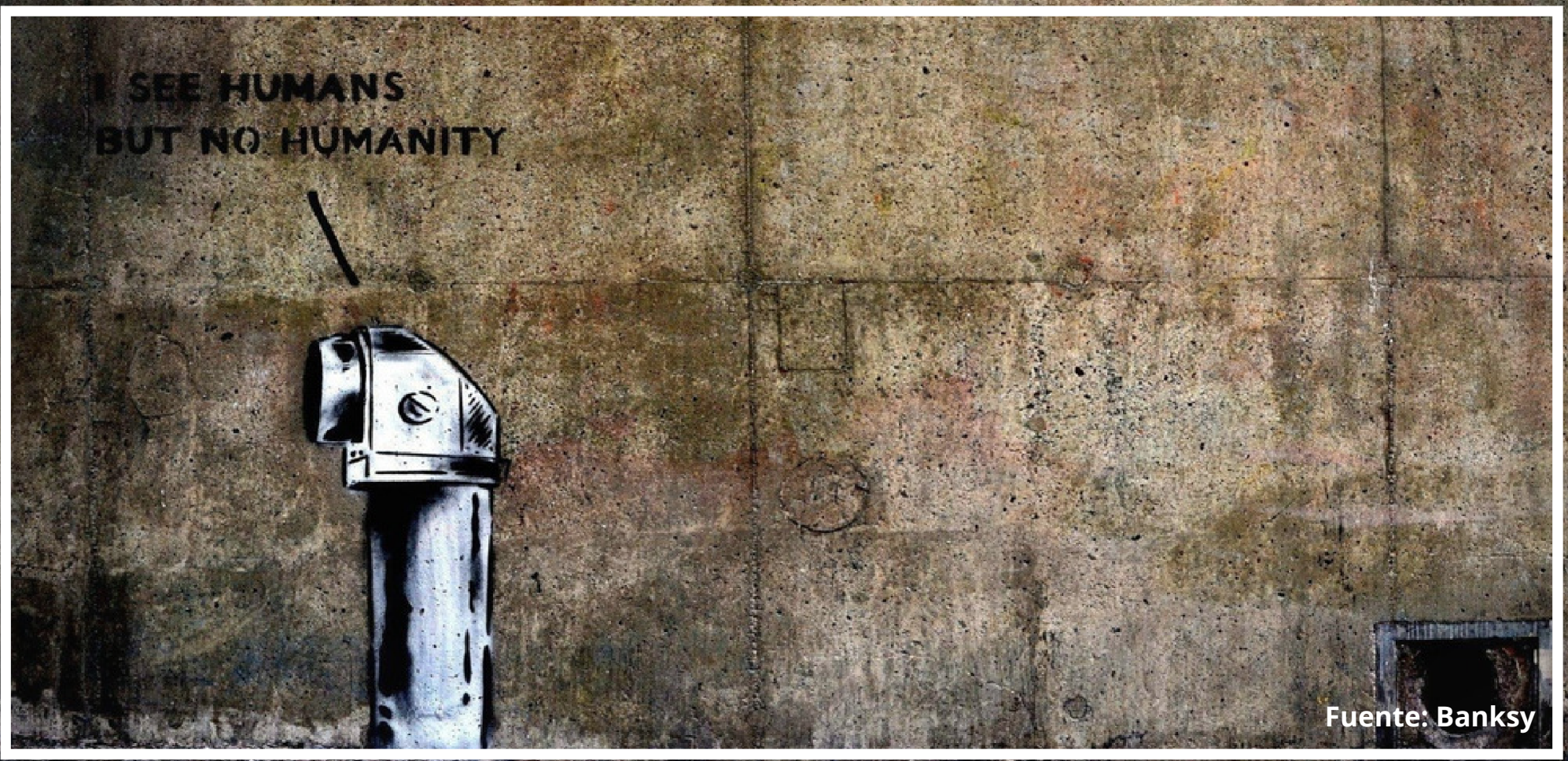

\title{
¿Somos cooperativos los humanos?
}

doi: $10.52749 /$ rh.v1i1.9

\section{DANIEL BARONA}

(iD) https://orcid.org/0000-0002-3339-243X

Licenciado en biología por la Universidad Nacional Agraria La Molina, egresado de la Maestría en Zoología por la Universidad Nacional Mayor de San Marcos. Docente de la Universidad Científica del Sur y divulgador científico que dirige los canales de El sueño de Darwin.

$\checkmark$ danielbarona1981@gmail.com

Resumen. La naturaleza humana incluye diversos atributos, como la competencia, la cooperación, el altruismo y el egoísmo. Muchas veces se le da más atención a los atributos negativos (egoísmo y competencia) que a los positivos (altruismo y cooperación). Sin embargo, la cooperación es un elemento vital para cualquier especie social, incluido el ser humano. En el presente trabajo se exploran los mecanismos que permiten la evolución de la cooperación en especies sociales, y se discuten las implicancias de tener individuos cooperadores y egoístas dentro de un grupo, a través de la teoría de juegos aplicada al comportamiento.

Palabras clave: altruismo, comportamiento, cooperación, competencia, egoísmo, evolución.

\section{Cómo citar este artículo:}

Barona, D. (2021). ¿Somos cooperativos los humanos? Futuro Hoy, 1(1), 49-52. https://doi.org/10.52749/rh.vli1.9

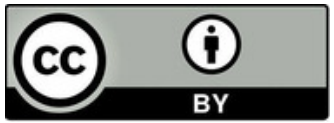




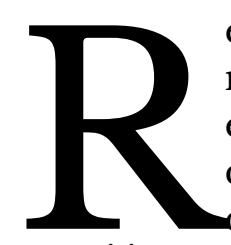

esulta casi intuitivo pensar en que la naturaleza humana es esencialmente egoísta y competitiva, y que nuestra trucción social para la cual se requiere establecer un tipo de contrato social tácito entre los miembros de la sociedad. Sin embargo, la realidad es totalmente distinta: si bien la competencia y el egoísmo son parte de nuestra naturaleza, en realidad prima la cooperación y, bajo ciertas circunstancias, el altruismo. En cualquier especie social la cooperación es vital para lograr la cohesión del grupo y para maximizar los beneficios que recibe cada individuo (Rand \& Nowak, 2013). Cooperando ganan todos, aunque siempre se le puede «hacer trampa» al sistema a través del engaño y las actitudes egoístas (Earley, 2010; Riehl \& Frederickson, 2016).

A lo largo de la historia se ha intentado explicar los fenómenos de cooperación y competencia propios de la especie humana, aunque para ello se ha utilizado siempre un enfoque netamente filosófico, social y/o religioso. Sin embargo, las explicaciones biológicas provenientes de la ciencia recién empiezan a darse de forma extensa desde Darwin (Darwin, 1871), aunque la investigación rigurosa siguiendo los métodos científicos modernos comenzó algunas décadas después del trabajo del propio Darwin.

En el presente trabajo se revisarán algunos mecanismos biológicos que favorecen la evolución de la cooperación en las especies. Asimismo, se revisarán y discutirán algunas de las conclusiones que surgen al considerar la teoría de juegos matemática aplicada al comportamiento.

Los mecanismos de la evolución de la cooperación

El biólogo matemático Martin Nowak ha propuesto cinco mecanismos que explican de forma matemática la evolución de la cooperación. El primero es la selección de parentesco (Hamilton, 1964; Nowak, 2006, 2012), es decir, el hecho de los individuos tienden a beneficiar con mayor frecuencia $y$ probabilidad a sus familiares. Mientras mayor sea el grado de parentesco, mayor será la tendencia a beneficiarlos, incluso produciéndose un acto verdaderamente altruis- ta -beneficiar a otro sin esperar nada a cambio-. El segundo mecanismo es la reciprocidad directa (Mailath \& Samuelson, 2006; Nowak, 2006, 2012), que consiste en que dos individuos cooperan mutuamente debido a que ambos se dan cuenta del beneficio que pueden recibir en el futuro si es que ayudan a un compañero, el cual luego puede retribuir el favor. El tercer mecanismo es la reciprocidad indirecta (Nowak \& Sigmund, 2005; Nowak, 2006, 2012), la cual se produce de manera que un individuo ayuda otro, pero el que lo retribuye es un tercero (un testigo). De esta manera, la reciprocidad indirecta funciona en base a la reputación que gana un individuo cooperador dentro de un grupo. El cuarto mecanismo es la reciprocidad de red (Tarnita et al., 2009; Nowak, 2006, 2012), en la que dentro de un grupo se forman redes de cooperadores que excluyen a otros individuos egoístas, y finalmente, el quinto y último mecanismo es la selección de grupo (Traulsen \& Nowak, 2006; Nowak, 2006, 2012), en la que grupos con una mayor proporción de cooperadores desplazan a grupos con una mayor proporción de egoístas.

Los mecanismos planteados por Nowak requieren de algunos ingredientes básicos: sistemas bioquímicos y/o sensoriales de reconocimiento de conespecíficos, así como una cognición compleja (Brosnan et al., 2010). Si bien esta última no es un requisito para la cooperación, sí permite incrementar la complejidad de las dinámicas sociales cooperativas, siendo un buen ejemplo de ello el ser humano. En nuestra especie incluso hay un elemento que puede potenciar el tercer mecanismo de reciprocidad indirecta: el lenguaje. Con el lenguaje humano la reputación de un individuo no solo se gana a través de la mirada atenta de sus compañeros, sino también a través del chisme y las habladurías. Estas últimas ayudan a que la reputación de los cooperadores se incremente rápida y efectivamente aun sin necesidad de que aquellos que transmiten oralmente las cualidades del cooperador hayan sido testigos visuales de los actos de cooperación (Nowak \& Highfield, 2012).

La empatía como ingrediente de la cooperación Relacionado a la cognición, existe la capacidad que tienen los individuos de muchas especies para «ponerse en los zapatos» de otro conespe- 
cífico. A esta capacidad se le conoce como empatía, y ocurre en diferentes especies animales, variando en grado entre ellas (De Waal, 2003; De Waal et al., 2006; De Waal, 2010). La empatía cognitiva, por un lado, consiste en que un individuo puede reconocer el estado de ánimo o emoción de otro individuo. En otras palabras, uno puede «predecir» qué está sintiendo el otro, aún a pesar de que esa emoción sea distinta a la propia en ese mismo instante. La empatía emocional, por otro lado, consiste en que un individuo es capaz de ver reflejadas las emociones de otro en sí mismo. Por ejemplo, si vemos a una persona triste, automáticamente esa tristeza o melancolía se refleja en nuestro estado de ánimo (Smith, 2006). Estos tipos de empatía son cruciales para poder cooperar con otros de manera adecuada, particularmente para especies en las que es vital el reconocimiento de rostros y posturas corporales antes de la interacción entre individuos.

El primatólogo holandés Frans de Waal plantea que la empatía es vital para la cooperación. De hecho, no es la especie humana la única que presenta empatía, sino que existen numerosas especies capaces de tal capacidad. El detalle es que la empatía puede presentarse en diferentes niveles de complejidad. De Waal argumenta que los niveles de empatía más complejos contienen a los más básicos, como si fueran muñecas rusas (De Waal, 2010). De este modo, existen tres niveles en orden creciente de complejidad: el contagio emocional o empatía emocional, la empatía cognitiva situacional relacionada a la preocupación por otros, y la empatía cognitiva atribucional en la que se adopta plenamente la perspectiva hacia el otro. Muchos animales poseen la empatía de primer nivel y muchos otros incluso la de segundo nivel, pero la de tercer nivel está presente en una cantidad mucho más reducida de animales, como por ejemplo los chimpancés, los elefantes, los delfines, y otras especies de mamíferos y aves. Los individuos que forman parte de una especie cooperarán más entre sí en el caso que puedan detectar y comprender las emociones de sus compañeros y entender que las necesidades del otro pueden ser similares o incluso distintas a las propias.
Comportamiento cooperativo y teoría de juegos

Llegados a este punto puede que también se nos pase por la mente la siguiente pregunta: si somos una especie de cooperadores, ¿̇por qué vemos muchas acciones egoístas y mucha competencia en las sociedades humanas? La respuesta a esta interrogante nos la puede dar la teoría de juegos.

La teoría de juegos es un área de las ciencias matemáticas aplicadas a la biología que trata de predecir cómo se comportará un individuo en respuesta al comportamiento de los demás compañeros (Broom \& Rychtár, 2013). Si dos individuos interactúan y ambos cooperan entre sí, probablemente seguirán cooperando en el futuro. Pero si uno de ellos coopera y el otro no, entonces la interacción entre ambos probablemente estará destinada a romperse o a llegar a una situación de desconfianza mutua. En esta dinámica de interacciones repetidas en el tiempo, lo más conveniente para el individuo (y para el grupo) es la cooperación. De ese modo se benefician ambas partes. Sin embargo, siempre quedará un nicho para el egoísta y el aprovechador. Un famoso juego llamado «Halcones y Palomas» plantea que dada la interacción entre individuos con actitud agresiva (actitud Halcón) e individuos con actitud pasiva (actitud Paloma), los individuos Halcón ganarán la disputa (por recursos, territorio, pareja reproductiva, etc.) a los individuos Paloma (Wilson, 2020). Sin embargo, a pesar de que aparentemente los individuos Paloma tenderían a desaparecer debido a que siempre están condenados a perder cuando se encuentren con un individuo Halcón, en realidad ambas estrategias coexistirán en una cierta proporción de equilibrio: una sociedad conformada en su totalidad por Halcones sería una sociedad tremendamente inestable en la que ningún individuo cooperará con otro, sino que todos velarán por su propio bienestar y lo harán sin importar los medios. Por el contrario, una sociedad conformada en su totalidad por Palomas sería una sociedad tan pacífica y utópica que bastaría que aparezca un único aprovechador egoísta y agresivo (individuo Halcón) para que explote a los demás con éxito; esto se traduciría en que la actitud Halcón empezaría a aumentar en frecuencia (ya que en realidad Halcón y Paloma son estrategias de comportamiento que pueden adoptar los indi- 
viduos en diferentes momentos y situaciones, aunque también hay cierta predisposición genética a que ciertos individuos tiendan a actuar más como halcones y otros como Palomas) (McNamara \& Leimar, 2020). La estrategia de ser Halcón cuando no hay muchos Halcones es muy tentadora y beneficiosa individualmente. Pero empieza a ser contraproducente a medida que la sociedad se va llenando de Halcones. De ahí que siempre se tienda a una proporción de equilibrio (Hoffman et al., 2016).

Del mismo modo, una sociedad compuesta por egoístas y cooperadores tenderá a una proporción de equilibrio, ya que una sociedad con mayoría de egoístas sería inestable, mientras que una sociedad con mayoría de cooperadores sería el sustrato ideal para que unos pocos egoístas se aprovechen con éxito de los cooperadores, aunque la estrategia de ser permanentemente egoísta no es una buena apuesta, dado que el egoísta persistente será fácilmente excluido del grupo.

\section{Referencias}

Broom, M., \& Rychtár, J. (2013). Game-theoretical models in biology. CRC Press.

Brosnan, S. F., Salwiczek, L., \& Bshary, R. (2010). The interplay of cognition and cooperation. Philosophical Transactions of the Royal Society B: Biological Sciences, 365(1553), 2699-2710. https://doi.org/10.1098/rstb.2010.0154

Darwin, C.R. (1871). The Descent of Man and Selection in Relation to Sex. Murray

De Waal, F. (2003). On the possibility of animal empathy. In Feelings and emotions: The Amsterdam symposium (pp. 379-99). Cambridge University Press.

De Waal, F. (2010). The age of empathy: Nature's lessons for a kinder society. Broadway Books.

De Waal, F., Macedo, S. E., \& Ober, J. E. (2006). Primates and philosophers: How morality evolved. Princeton University Press.

Earley, R. L. (2010). Social eavesdropping and the evolution of conditional cooperation and cheating strategies. Philosophical Transactions of the Royal Society B: Biological Sciences, 365(1553), 2675-2686. https://doi.org/10.1098/rstb.2010.0147

Hamilton, W.D. (1964). The genetical evolution of social behaviour, I and II. J. Theor. Biol. 7, 1-52.

Hoffman, M., Yoeli, E., \& Navarrete, C. D. (2016). Game theory and morality. In The evolution of morality (pp. 289-316). Springer, Cham.

Mailath, G.J., Samuelson, L. (2006). Repeated Games and Reputations: long-run Relationships. Oxford University Press.

McNamara, J. M., \& Leimar, O. (2020). Game theory in biology: concepts and frontiers. Oxford University Press.

\section{Conclusiones}

Las especies gregarias o sociales como la nuestra necesitan de la cooperación. Sin embargo, algunos individuos aprovecharán las oportunidades que puedan presentarse para explotar a otros en beneficio propio. Por supuesto, en qué medida y de qué forma ocurre esto último en nuestra especie dependerá también de factores socioculturales y económicos. Aún con todo, los cimientos de la interacción humana y de las estructuras sociales, culturales y económicas dependen en gran medida de que los individuos que conformamos las sociedades cooperemos los unos con los otros de diversas maneras, ya sea colaborando para sacar adelante una empresa o aceptando los consensos universales existentes en nuestra sociedad global, como por ejemplo, aquellos relacionados al valor que tiene el dinero como moneda de cambio, entre otros.

Nowak M.A., Highfield, R. (2012). Supercooperadores. Grupo Zeta.

Nowak, M. A. (2006). Five Rules for the Evolution of Cooperation. Science, 314(5805), 1560-1563. https://doi.org/10.1126/science.1133755

Nowak, M. A. (2012). Evolving cooperation. Journal of theoretical biology, 299(0), 1-8

Nowak, M.A., Sigmund, K. (2005). Evolution of indirect reciprocity. Nature 427, 1291-1298

Rand, D. G., \& Nowak, M. A. (2013). Human cooperation. Trends in Cognitive Sciences, 17(8), 413-425. https://doi.org/10.1016/j.tics.2013.06.003

Riehl, C., \& Frederickson, M. E. (2016). Cheating and punishment in cooperative animal societies: Table 1. Philosophical Transactions of the Royal Society B: Biological Sciences, 371(1687), 20150090. https://doi.org/10.1098/rstb.2015.0090

Smith, A. (2006). Cognitive Empathy and Emotional Empathy in Human Behavior and Evolution. Psychol Rec 56, 3-21. https://doi.org/10.1007/BF03395534

Tarnita, C.E., Antal, T., Ohtsuki, H., Nowak, M.A. (2009). Evolutionary dynamics in set structured populations. Proc. Natl. Acad. Sci,106, 8601-8604

Traulsen, A., \& Nowak, M. A. (2006). Evolution of cooperation by multilevel selection. Proceedings of the National Academy of Sciences, 103(29), https://doi.org/10.1073/pnas.0602530103

Wilson, M. L. (2020). Hawks, Doves, and mongooses. Proceedings of the National Academy of Sciences, 202021188. https://doi.org/10.1073/pnas.2021188117 